\title{
APROTININ IN PRIMARY VALVE REPLACEMENT AND RECONSTRUCTION: A MULTICENTER, DOUBLE-BLIND, PLACEBO-CONTROLLED TRIAL.
}

Michael N. D'Ambra, MD

Cary W. Akins, MD

Eugene H. Blackstone, MD

Sharon L. Bonney, MD

Lawrence H. Cohn, MD

Delos M. Cosgrove, MD

Jerrold H. Levy, MD

Karen E. Lynch, BSN

Rosemarie Maddi, MD
Background: Patients having cardiac operations often require blood transfusions. Aprotinin reduces the need for blood transfusions during coronary artery bypass graft operations. To determine the safety and effectiveness of aprotinin in reducing the use of allogeneic blood and postoperative mediastinal chest tube drainage, we studied 212 patients undergoing primary sternotomy for valve replacement or repair. Methods: This study was multicenter, randomized, prospective, double-blind, and placebo-controlled. Patients received highdose aprotinin $(n=71)$, low-dose aprotinin $(n=70)$, or placebo $(n=71)$. The study medication was given as a loading dose followed by a continuous infusion and pump prime dose. Heparin administration was standardized. Transfusions, postoperative mediastinal shed blood, and adverse events were tracked. Results: Demographic profiles were similar among the treatment groups. Aprotinin did not decrease the percentage of patients receiving transfusions when compared with placebo (high-dose aprotinin, $63 \%, p=0.092$; low-dose aprotinin, $52 \%, p=0.592$; placebo, $48 \%$ ). Aprotinin was associated with a reduction in the volume of mediastinal shed blood (high-dose aprotinin vs placebo, $p=0.002$; low-dose aprotinin vs placebo, $p=0.017$ ). Adverse events were equally distributed among the treatment groups except for postoperative renal dysfunction (high-dose aprotinin, 11\%; low-dose aprotinin, 7\%; placebo, $0 \% ; p=0.01$ ). A disproportionate number of patients in the high-dose aprotinin group with postoperative renal dysfunction also had diabetes mellitus. Conclusions: Aprotinin treatment in this population did not reduce allogeneic blood use, although there were significant reductions in the volume of mediastinal shed blood. (J Thorac Cardiovasc Surg 1996;112:1081-9)
C ardiac operations necessitating extracorporeal circulation often are associated with the use of allogeneic blood for the treatment of bleeding or to counter the effects of hemodilution. ${ }^{1}$ Exposure to allogeneic blood has decreased over the past 10

From the Departments of Anesthesia and Surgery, Massachusetts General Hospital, Boston, Mass.; the Department of Surgery, University of Alabama, Birmingham, Ala.; Miles Inc., West Haven, Conn.; the Departments of Anesthesia and Surgery, Brigham and Women's Hospital, Boston, Mass.; the Department of Surgery, The Cleveland Clinic Foundation, Cleveland, Ohio; and the Department of Anesthesia, Emory University Hospital, Atlanta, Ga.

Research supported in part by grants from Miles Inc., West Haven, Conn., and Rowland Foundation, Cambridge, Mass.

Received for publication Oct. 19, 1995; revisions requested Dec. 26, 1995; revisions received May 3, 1996; accepted for publication May 6, 1996.

Address for reprints: Michael N. D'Ambra, MD, Cardiac Anesthesia Group, Massachusetts General Hospital, Boston, MA 02114-2696.

Copyright $(1996$ by Mosby-Year Book, Inc.

$0022-5223 / 96 \$ 5.00+0 \quad \mathbf{1 2} / \mathbf{1} / 74706$ years with the appearance of cell salvaging and reinfusion techniques, a better understanding of the limits of anemia, and expansion of predonation autologous blood programs. ${ }^{2}$ Despite these advances, patients continue to be exposed to allogeneic blood. In a study by Hardy and associates ${ }^{2}$ that included 158 patients undergoing primary operations for valve repair, patients received transfusions of $3.0 \pm 2.7$ units (mean \pm standard deviation) of packed red blood cells during their hospitalizations. Until recently, most pharmacologic means of reducing postoperative bleeding after cardiac operations produced mixed results. ${ }^{1,3-5}$

Aprotinin, a serine protease inhibitor with potent inhibitory effects on kallikrein and plasmin, is a singlechain polypeptide consisting of 58 amino acid residues and three disulfide bridges, with a molecular weight of 6512 daltons. Aprotinin was identified in 1930 by Kraut and colleagues as a kallikrein inactivator in bovine lymph nodes and in 1936 by Kunitz and Northrop as a trypsin inhibitor in the bovine pancreas. ${ }^{6}$ The activity of aprotinin is expressed in kallikrein 
inactivator units (KIU), with one KIU defined as that amount of aprotinin that decreases the activity of two biologic kallikrein units by $50 \%$. One milligram of aprotinin is equivalent to $7143 \mathrm{KIU}$.

In 1987 Royston and associates ${ }^{7}$ reported a significant reduction in the postoperative mediastinal blood loss and in the use of blood transfusions when aprotinin was administered prophylactically to patients requiring cardiopulmonary bypass. Before that time the therapeutic uses for aprotinin were based on its ability to inhibit trypsin, plasmin, and tissue kallikrein and included acute pancreatitis, shock syndromes, and hyperfibrinolytic hemorrhage. . $11^{-11}$

A whole-body inflammatory response is seen when the blood contacts foreign surfaces during cardiopulmonary bypass. ${ }^{12}$ This stress response is mediated in part by activation of kallikrein and plasmin..$^{13}$ Aprotinin inhibits kallikrein and plasmin in concentrations of $200 \mathrm{KIU} / \mathrm{ml}$ and $50 \mathrm{KIU} / \mathrm{ml}$, respectively, and may attenuate the deleterious effects of the stress response by multiple mechanisms. ${ }^{13}$ The purpose of the current dose-response study was to determine whether aprotinin therapy could safely and effectively reduce exposure to allogeneic blood in patients undergoing primary sternotomy for valve replacement or repair. A kallikrein-inhibiting dose, the high-dose aprotinin group, and a nonkallikrein-inhibiting dose, the lowdose aprotinin group, were included to investigate the blood-sparing effect of aprotinin.

\section{Methods}

Study design. This was a multicenter, randomized, double-blind, placebo-controlled, parallel-group study conducted at five sites (23 surgeons). Written informed consent was obtained from all patients according to the guidelines of each hospital's institutional review board. Patients who qualified for study entry were randomized to receive aprotinin, in one of two doses, or placebo.

A separate random code, using blocks of six, was generated for each site by the statistical department of Bayer Inc. (SAS software, SAS Institute, Inc., Cary, N.C.). Doubleblind conditions were maintained throughout the study.

Patient selection and demographics. Any patient with a primary diagnosis of valvular heart disease who met all of the inclusion criteria and none of the exclusion criteria were eligible to enter the study. The inclusion criteria included primary aortic and/or mitral valve replacement or repair through a median sternotomy. The patients included both men and women who were 18 years of age or older.

Patients were not eligible for participation if they had a known or suspected allergy to aprotinin, a history of bleeding diathesis or known coagulation factor deficiency that would require prophylactic use of drugs to improve hemostasis, were scheduled to undergo a concurrent coronary artery bypass graft operation or other cardiac operation, were participants in any other investigational drug trial, or refused allogeneic blood transfusion therapy.
A total of 213 patients were enrolled and randomized at five sites (site $1=43$ patients; site $2=43$ patients; site $3=$ 44 patients; site $4=40$ patients; and site $5=42$ patients). A total of 212 patients received study medication and were included in the safety analysis (high-dose aprotinin, $n=71$; low-dose aprotinin, $n=70$; placebo, $n=71$ ). Of these 212 patients, 191 were included in the primary analysis of efficacy (high-dose aprotinin, $n=65$; low-dose aprotinin, $n=62$; placebo, $n=64$ ).

Study medication. A preinfusion test dose of study medication was administered after induction of anesthesia. If no untoward effects of the study medication were noted, an intravenous loading dose (280 $\mathrm{mg}$ aprotinin or $2 \times 10^{6}$ KIU aprotinin $=$ high-dose aprotinin; $140 \mathrm{mg}$ aprotinin or $1 \times 106 \mathrm{KIU}$ aprotinin = low-dose aprotinin; normal saline solution $=$ placebo) was infused over 20 to 30 minutes followed by a continuous infusion $(70 \mathrm{mg}$ or $500,000 \mathrm{KIU} / \mathrm{hr}$ aprotinin $=$ high-dose aprotinin; $35 \mathrm{mg}$ or $250,000 \mathrm{KIU} / \mathrm{hr}$ aprotinin = low-dose aprotinin; normal saline solution $=$ placebo), which was infused until chest closure. An additional dose of aprotinin or placebo, equivalent to the loading dose, was added to the pump prime.

Aprotinin was supplied by Bayer Inc. (West Haven, Conn.) in a concentration of $1.4 \mathrm{mg} / \mathrm{ml}(10,000 \mathrm{KIU} / \mathrm{ml})$ in $0.9 \%$ sodium chloride solution, without additives or preservative substances. The placebo $(0.9 \%$ sodium chloride solution) was also provided by Bayer Inc. The high-dose aprotinin regimen used in this study was the "Hammersmith regimen," as described by Royston. ${ }^{13}$ The study medication for each patient was supplied in a case pack containing 14 vials. The loading dose vials, pump prime vials, and constant infusion vials were separately identified and packaged within the pack for each patient. Investigators were blinded to the identity and lot number of each case pack.

Procedures. Anesthesia, conduct of cardiopulmonary bypass, the operation, and postoperative intensive care procedures were those routinely practiced at each study center, including monitoring of the activated clotting time with either kaolin or celite activation. Because of the interference of aprotinin with celite activation, heparin anticoagulation was standardized to either a bolus of heparin of $300 \mathrm{U} / \mathrm{kg}$ or more with a subsequent heparin dose of $150 \mathrm{U} / \mathrm{kg} 90$ minutes later if the patient was still on cardiopulmonary bypass or as predicted by a heparin dose-response test. ${ }^{14}$

Blood conservation measures included the reinfusion of residual blood from the cardiotomy reservoir and in $79 \%$ of the patients the reinfusion of postoperative mediastinal shed blood. Efforts to standardize the use of allogeneic blood were established. During cardiopulmonary bypass, packed red blood cells were administered if the hematocrit value fell below 18\%; after the operation, packed red blood cells were given if the hematocrit value was less than $21 \%$. Patients who had donated their own blood before the operation received this blood after the operation. Blood products were administered when the patient's clinical condition necessitated transfusion. Epsilon-aminocaproic acid and desmopressin were used prophylactically only to treat active bleeding after heparin reversal, and their use was documented. 
Study end points. The effectiveness of aprotinin was assessed by the percentage of patients requiring any allogeneic blood transfusions and by the number of units of allogeneic blood required per patient. The use of allogeneic blood including the use of whole blood, packed red blood cells, fresh frozen plasma, platelets, and cryoprecipitate were tracked according to the time when they were administered: during the operation, during the first 24 hours after the operation, and from postoperative day 2 through hospital discharge. The ability of aprotinin to reduce postoperative mediastinal chest tube drainage was assessed through the volume of shed blood during the first 12 hours after transfer to the intensive care unit and for the interval from 12 to 24 hours or until the thoracic drains were removed. The frequency of and reason for reoperation for bleeding were tracked.

Patients who could not be evaluated for efficacy. Excluded from the efficacy analysis were the following categories of patients: patients who did not receive the full dose of study medication $(n=3)$; patients who died within the first 6 hours after the operation $(n=1)$; patients who required reexploration for excessive bleeding from an anatomically localized site $(n=7)$; patients who had myocardial dysfunction necessitating intraaortic balloon counterpulsation at the end of the operation $(n=1)$; patients who had major bleeding after the operation from sites unrelated to the surgical procedure $(n=2)$; patients who received allogeneic blood products in the prime of the cardiopulmonary bypass circuit or before the operation after the sample for the preoperative complete blood count was drawn $(n=4)$; patients who got study medication but did not then have a cardiac operation with cardiopulmonary bypass $(n=2)$; and patients who had a prior median sternotomy $(n=1)$. All patients were included in the safety analysis.

Statistical methods. In the design of the study it was estimated that $60 \%$ of patients given placebo would require allogeneic blood. It was specified that a $50 \%$ reduction would be considered clinically meaningful. Sample size calculations determined that with 65 patients per treatment group using a two-tailed test with an $\alpha$ level of 0.05 , one would have more than $90 \%$ power of detecting a difference between the high-dose aprotinin group and placebo. All statistical tests were performed with SAS Institute statistical software.

All statistical tests were two-tailed and were performed with an $\alpha$ level of 0.05 . The primary efficacy variable was the percentage of patients requiring any allogeneic blood transfusions. The secondary efficacy variable was the number of units of allogeneic blood required per patient. Intent-to-treat analyses were performed on all patients given study medication who had operations involving cardiopulmonary bypass. The primary comparison in each analysis was that of high-dose aprotinin versus placebo. Pairwise comparisons of low-dose aprotinin versus placebo and high-dose aprotinin versus low-dose aprotinin were also performed. The primary efficacy variable and categoric variables other than treatment emergency adverse event rates and incidence rates of laboratory abnormalities were analyzed by means of the Mantel-Haenszel test. Confidence intervals for the difference in proportions between treatment groups were calculated by MantelHaenszel methods.
Because of gross departures from normality in the units of allogeneic blood required and the number of milliliters of allogeneic blood required, the null hypothesis of no treatment difference was tested by means of a two-way analysis of variance of ranked data, with the data ranked over all the centers. A standard two-way analysis of variance model was used for all other continuous variables. The analysis of variance model included the effects of drug, center, and drug-by-center interaction. If no significant drug-by-center interaction was observed, the main effect's model was used. When a standard analysis of variance model was used, least square means for each treatment and 95\% confidence intervals (CI) for the pairwise treatment differences were calculated. No confidence intervals were presented when the ranked data were analyzed because of difficulty in interpretation. In this case, however, the least square means reported were calculated with the use of the untransformed data.

To investigate the effect of the patient's baseline characteristics on the probability of getting a transfusion, we pooled the data across treatment groups and analyzed them using a logistic regression model with age, height, weight, and red blood cell mass as independent variables. A term for center was always included in the model. Oniy age and red blood cell mass were identified as independent predictors. A final logistic regression model was run to test the hypothesis of no treatment effect after adjusting for center and for differences in age and preoperative red blood cell mass.

Safety was assessed through the frequency distribution of adverse events reported by the site investigator. Adverse events were categorized by similar diagnosis. No clinical parameters were established for the diagnosis of adverse events. Renal dysfunction was categorically defined as acute renal failure, renal failure, renal function abnormality, and renal tubular necrosis. Myocardial dysfunction was categorically defined as congestive heart failure and heart failure or both. Adverse events affecting the cardiovascular system included atrial and ventricular arrhythmias, hypotension, myocardial infarction, heart failure, and congestive heart failure. The frequency distribution of adverse events, as defined by the investigators, was analyzed by means of either Fisher's exact test or a $\chi^{2}$ test. Fisher's exact tests were used if at least $25 \%$ of the cells had expected values less than five; otherwise, $\chi^{2}$ tests were used.

\section{Results}

Population. There were no significant differences among the treatment groups in demographic characteristics, preoperative hemoglobin values, preoperative prothrombin time and partial thromboplastin time, preoperative creatinine concentration, history of previous myocardial infarction, type of valvular lesion present, types of operative procedure performed, history of hypertension, preoperative left ventricular ejection fraction, and preoperative New York Heart Association functional class (Table I).

The intraoperative course was similar for all groups, including total crossclamp time, duration of the operation, and total anesthesia time (Table II). 
Table I. Demographic summary

\begin{tabular}{|c|c|c|c|}
\hline & $\begin{array}{l}\text { High-dose } \\
\text { aprotinin }\end{array}$ & $\begin{array}{l}\text { Low-dose } \\
\text { aprotinin }\end{array}$ & Placebo \\
\hline No. of patients & 65 & 62 & 64 \\
\hline Age (yr) & $59.8 \pm 3.1$ & $59.2 \pm 3.2$ & $60.0 \pm 3.1$ \\
\hline Men (\%) & 48 & 53 & 47 \\
\hline White $(\%)$ & 95 & 90 & 95 \\
\hline Weight (kg) & $71.8 \pm 3.9$ & $72.0 \pm 4.1$ & $72.5 \pm 4.0$ \\
\hline Hypertension (\%) & 46 & 29 & 36 \\
\hline Pulmonary hypertension (\%) & 34 & 47 & 40 \\
\hline MI history (\%) & 12 & 3 & 8 \\
\hline NYHA class & $2.6 \pm 0.01$ & $2.7 \pm 0.01$ & $2.7 \pm 0.01$ \\
\hline Baseline PT (mg/dl) & $11.8 \pm 12.3$ & $12.0 \pm 11.6$ & $11.9 \pm 10.9$ \\
\hline BAseline PTT (mg/dl) & $31.4 \pm 6.3$ & $31.4 \pm 6.8$ & $29.6 \pm 6.3$ \\
\hline BAseline $\mathrm{Hgb}$ (mg/dl) & $13.5 \pm 0.04$ & $13.6 \pm 0.04$ & $13.5 \pm 0.04$ \\
\hline Baseline creatinine (mg/dl) & $1.10 \pm 0.02$ & $1.12 \pm 0.02$ & $1.10 \pm 0.02$ \\
\hline Aortic stenosis (\%) & 52 & 34 & 43 \\
\hline Aortic insufficiency $(\%)$ & 34 & 34 & 30 \\
\hline Mitral stenosis (\%) & 18 & 24 & 21 \\
\hline Mitral insufficiency (\%) & 34 & 53 & 51 \\
\hline
\end{tabular}

The total bypass time was significantly different between the low-dose aprotinin group and the placebo group (CI: difference between low-dose aprotinin and placebo, $2.9 \%$ to $25.7 \% ; p=0.014$ ).

Transfusion prevalence. Few patients participated in autologous predonation programs. There was no difference between the treatment groups in the percentages of patients predonating their blood (high-dose aprotinin, 5\%; low-dose aprotinin, 6\%; placebo, $5 \%$ ).

Aprotinin, high-dose and low-dose, did not significantly reduce the percentage of patients requiring allogeneic transfusions. The percentages of patients who required allogeneic red blood transfusions (packed red blood cells and whole blood) were 63\% $(41 / 65)$ in the high-dose aprotinin group, 52\% (32/62) in the low-dose aprotinin group, and 48\% (31/64) in the placebo group; none of the pairwise hypothesis tests reached statistical significance for the difference in proportions between the treatment groups (difference between high-dose aprotinin and placebo, $\mathrm{CI}=$ $-2.2 \%$ to $31.8 \%, p=0.092$; difference between lowdose aprotinin and placebo, $\mathrm{CI}=-11.6 \%$ to $20.8 \%$, $p=0.592$ ). Even when all patients who received study medication and were operated on were included (intent-to-treat analysis), there was no statistically significant difference in the percentage of patients receiving allogeneic blood transfusions between the aprotinin treatment groups and the placebo group (high-dose aprotinin group, $66 \%$ transfused; low-dose aprotinin group, $56 \%$ transfused; placebo, $53 \%$ transfused). The
Table II. Intraoperative course

\begin{tabular}{lccc}
\hline & $\begin{array}{c}\text { High-dose } \\
\text { aprotinin }\end{array}$ & $\begin{array}{c}\text { Low-dose } \\
\text { aprotinin }\end{array}$ & Placebo \\
\hline Total bypass time (min) & $102.1 \pm 4.0$ & $107.4 \pm 4.1$ & $93.1 \pm 4.0$ \\
Total crossclamp time (min) & $64.5 \pm 3.1$ & $67.3 \pm 3.2$ & $59.7 \pm 3.2$ \\
Total anesthesia time (hr) & $4.6 \pm 0.1$ & $4.5 \pm 0.1$ & $4.3 \pm 0.1$ \\
Duration of operation (hr) & $3.5 \pm 0.1$ & $3.5 \pm 0.1$ & $3.2 \pm 0.1$ \\
Aortic replacement or repair (\%) & 62 & 44 & 52 \\
Mitral replacement (\%) & 15 & 29 & 23 \\
Mitral repair (\%) & 15 & 15 & 19 \\
Tricuspid or pulmonic & 3 & 0 & 0 \\
$\quad$ replacement/repair (\%) & & & \\
Aortic and mitral & 5 & 10 & 3 \\
$\quad$ repair/replacement $(\%)$ & & & \\
Other combination (\%) & 0 & 3 & 3 \\
\hline
\end{tabular}

For continuous variables results are presented as mean \pm standard error.

confidence interval for the difference in proportions between the high-dose aprotinin group and the placebo group was $-3.0 \%$ to $29.3 \%(p=0.113)$ and for low-dose aprotinin and placebo groups, $-12.9 \%$ to $17.8 \%(p=0.763)$.

There were no statistically significant differences among the treatment groups in the mean number of units of allogeneic blood transfused (given as mean \pm standard error): red blood cells (high-dose aprotinin, $2.0 \pm 0.4$ units; low-dose aprotinin, $1.9 \pm$ 0.4 units; placebo, $1.3 \pm 0.4$ units), platelets (highdose aprotinin, $2 \pm 0.7$ units; low-dose aprotinin, $0.4 \pm 0.7$ units; placebo, $0.9 \pm 0.7$ units), fresh frozen plasma (high-dose aprotinin, $0.4 \pm 0.2$ units; low-dose aprotinin, $0.4 \pm 0.2$ units; placebo, $0.5 \pm$ 0.2 units), or cryoprecipitate (high-dose aprotinin, $0.2 \pm 0.2$ units; low-dose aprotinin, $0.2 \pm 0.2$ units; placebo, $0.3 \pm 0.2$ units).

The transfusion distribution in the high-dose aprotinin group was significantly different from those in the low-dose aprotinin and placebo groups. Forty-six percent of patients in the high-dose aprotinin group received intraoperative transfusions compared with $37 \%$ in the low-dose aprotinin group and $23 \%$ in the placebo group (CI: difference between high-dose aprotinin and placebo, $7 \%$ to $39 \%$, $p=0.006$; difference between low-dose aprotinin and placebo, $-0.9 \%$ to $30 \%, p=0.073$; Fig. 1). This difference among the treatment groups did not persist into the postoperative period. During the first 24 hours after the operation $15 \%$ of high-dose aprotinin group, $16 \%$ of the low-dose aprotinin group, and $28 \%$ of the placebo group received transfusions (CI: difference between high-dose aprotinin and placebo, $-26 \%$ to $1 \%$; difference between low-dose aprotinin and placebo, $-25 \%$ to $3 \%$ ). The transfusion prevalences were similar for 


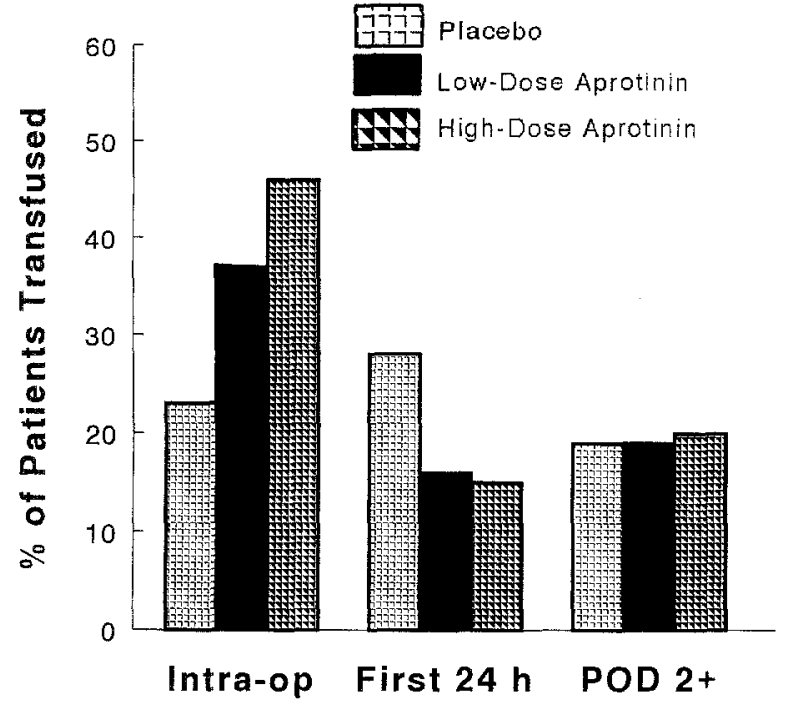

Fig. 1. The intraoperative rate of transfusion for patients treated with high-dose aprotinin was significantly higher than the rate of transfusion for patients in the low-dose aprotinin and placebo treatment groups $(p=0.03)$. This difference did not continue into the postoperative period $(p>0.05)$. POD, Postoperative day.

all groups during the interval from postoperative day 2 through discharge, with $20 \%$ of the high-dose aprotinin group, $19 \%$ of the low-dose aprotinin group, and $19 \%$ of the placebo group requiring allogeneic blood (CI: difference between high-dose aprotinin and placebo, $-12 \%$ to $14 \%$; difference between low-dose aprotinin and placebo, $-12 \%$ to $14 \%$ ).

A difference existed among the treatment groups when the number of units of blood was stratified. Eighteen percent of the high-dose aprotinin group received one unit of allogeneic blood whereas $3 \%$ of the low-dose aprotinin group and $9 \%$ of the placebo group received only one unit of allogeneic blood $(p=0.02)$. This difference did not persist when two or more units of allogeneic blood were transfused. Additionally, the use of allogeneic blood did not correlate with the mediastinal chest tube drainage in the high-dose aprotinin group (Fig. 2).

The percentages of patients who required allogeneic blood transfusions were also analyzed according to baseline characteristics including sex, age, and body weight (Table III). In each of these subcategories, as with the overall analysis, there was a trend toward an increased percentage of patients requiring allogeneic blood in the aprotinin groups.

Red cell blood volume. More patients in the high-dose aprotinin group than the other two treatment groups had preoperative red blood cell vol-

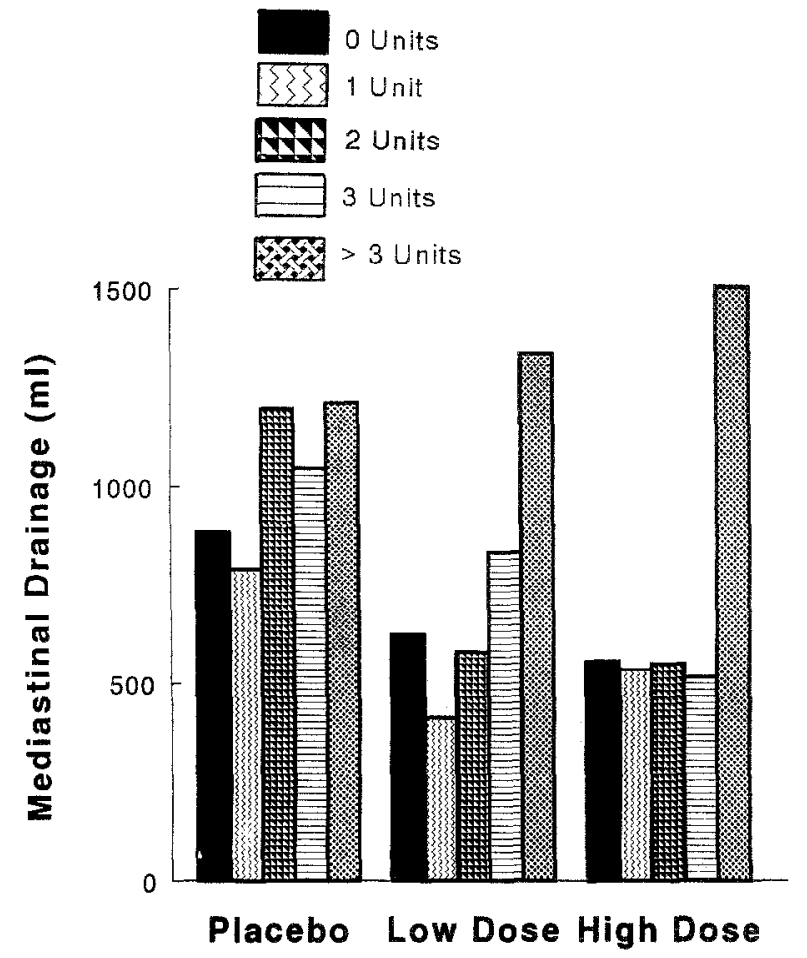

Fig. 2. Mediastinal drainage was not a predictor of the number of allogeneic red blood cells required in the high-dose aprotinin group. The different bars represent the number of units transfused.

umes less than or equal to $1600 \mathrm{ml}$ (high-dose aprotinin, 40\%; low-dose aprotinin, 34\%; placebo, $31 \%$ ). The $95 \%$ confidence interval for the difference between the high-dose aprotinin group and the placebo group in the proportion of such patients ranged from $-8 \%$ to $25 \%(p=0.305)$, whereas the $95 \%$ confidence interval for the difference between the low-dose aprotinin treatment group and the placebo group ranged from $-13 \%$ to $17 \%(p=$ 0.794). Of those patients with a preoperative red blood cell volume less than or equal to $1600 \mathrm{ml}$, $85 \%, 71 \%$, and $60 \%$ of the patients received transfusions of allogeneic red blood cells in the high-dose aprotinin, low-dose aprotinin, and placebo groups, respectively (high-dose aprotinin vs placebo, $p=$ 0.913 ; low-dose aprotinin vs placebo, $p=0.732$ ).

Postoperative mediastinal shed blood. The postoperative thoracic drainage rate was statistically significantly less in the high-dose aprotinin $(37 \mathrm{ml} /$ hr; $p=0.0001$ ) and in the low-dose aprotinin (46 $\mathrm{ml} / \mathrm{hr} ; p=0.0001$ ) treatment groups than in the placebo-treated patients $(71 \mathrm{ml} / \mathrm{hr})$ during the first 8 hours after the operation. The total postoperative thoracic drainage was reduced by $33 \%$ in the high- 
Table III. Proportion of patients requiring any allogenic blood, broken down by age, sex, and weight

\begin{tabular}{ccccc}
\hline \multicolumn{6}{c}{ Characteristic Subgroup } & $\begin{array}{c}\text { High-dose } \\
\text { aprotinin } \\
(n=65)\end{array}$ & $\begin{array}{l}\text { Low-dose } \\
\text { aprotinin } \\
(n=62)\end{array}$ & $\begin{array}{c}\text { Placebo } \\
(n=64)\end{array}$ \\
\hline \multirow{2}{*}{ Sex } & Male & $15 / 31(48 \%)$ & $11 / 33(33 \%)$ & $10 / 30(33 \%)$ \\
& Female & $26 / 34(76 \%)$ & $21 / 29(72 \%)$ & $21 / 34(62 \%)$ \\
Age & $<60 \mathrm{yr}$ & $12 / 29(41 \%)$ & $13 / 30(43 \%)$ & $10 / 30(33 \%)$ \\
& $\geq 60 \mathrm{yr}$ & $29 / 36(81 \%)$ & $19 / 32(59 \%)$ & $21 / 34(62 \%)$ \\
Weight & $<60 \mathrm{~kg}$ & $15 / 18(83 \%)$ & $9 / 13(69 \%)$ & $9 / 11(82 \%)$ \\
& $\geq 60 \mathrm{~kg}$ & $26 / 47(55 \%)$ & $23 / 49(47 \%)$ & $22 / 53(42 \%)$ \\
\hline
\end{tabular}

dose aprotinin group ( $p=0.002)$ and by $26 \%$ in the low-dose aprotinin group ( $p=0.017)$, as compared with the placebo group. In addition, there appears to be a trend toward a dose response to aprotinin in patients over the age of 59 years (high-dose aprotinin vs placebo, $p=0.0001$; low-dose aprotinin vs placebo, $p=0.0032$ ).

Treatment with other hemostatic drugs. The administration of therapeutic hemostatic agents, other than aprotinin, to control bleeding after cardiopulmonary bypass differed significantly among the treatment groups. The percentage of patients treated with epsilon-aminocaproic acid in the placebo group was $6 \%(4 / 64)$, whereas no patients in the aprotinin groups were treated with epsilonaminocaproic acid. This difference in the percentage of patients receiving epsilon-aminocaproic acid was significant for both aprotinin groups when compared with the placebo group $(p=0.05)$. Desmopressin therapy was given to $9 \%(6 / 65)$ of the high-dose aprotinin group, $8 \%(5 / 61)$ of the lowdose aprotinin group, and 19\% (12/64) of the placebo group (CI: difference between high-dose aprotinin and placebo, $-22 \%$ to $2 \%$; difference between low-dose aprotinin and placebo, $-22 \%$ to $1 \%$ ). However, the exclusion of patients treated with epsilon-aminocaproic acid alone, desmopressin alone, or both, did not affect the difference in transfusion requirement between the aprotinin and placebo groups.

Hemoglobin. The percentage change in hemoglobin value from the preoperative baseline value to postoperative day 7 was statistically significant for the high-dose aprotinin group compared with the placebo and low-dose aprotinin groups. The changes in hemoglobin from the baseline value to the postoperative value were a decrease of $2.46 \mathrm{gm} / \mathrm{dl}$ for the high-dose aprotinin group, $3.29 \mathrm{gm} / \mathrm{dl}$ for the lowdose aprotinin group, and $3.29 \mathrm{gm} / \mathrm{dl}$ for the placebo group (CI: difference between high-dose aprotinin and placebo, -1.43 to $-0.23, p=0.007$; difference
Table IV. Treatment of emergency events categorically defined as renal dysfunction

\begin{tabular}{lcccc}
\hline \multicolumn{1}{c}{ Event } & $\begin{array}{c}\text { High-dose } \\
\text { aprotinin } \\
(n=71)\end{array}$ & $\begin{array}{c}\text { Los-dose } \\
\text { aprotinin } \\
(n=70)\end{array}$ & $\begin{array}{c}\text { Placebo } \\
(n=71)\end{array}$ & $\begin{array}{c}\text { Overall } \\
(n \text { value }\end{array}$ \\
\hline Renal function abnormal & 6 & 0 & 0 & 0.004 \\
Renal failure & 1 & 4 & 0 & 0.049 \\
Renal tubular necrosis & 1 & 0 & 0 & 1.000 \\
Acute kidney failure & 0 & 1 & 0 & 0.330 \\
\hline
\end{tabular}

between high-dose aprotinin and low-dose aprotinin and placebo, -1.43 to $-0.22, p=0.008$ ) as a result of the higher intraoperative transfusion rates and the reduction in postoperative shed mediastinal drainage in the aprotinin-treated groups.

Safety. A total of 212 patients were eligible for the safety analysis: 71 in the high-dose aprotinin group, 70 in the low-dose aprotinin group, and 71 in the placebo group.

The overall incidence rates for reported adverse events in the high-dose aprotinin group, the lowdose aprotinin group, and the placebo group were not significantly different $(p=0.838)$. Seventy-six percent of the high-dose aprotinin group, $73 \%$ of the low-dose aprotinin group, and $72 \%$ of the placebo group reported one or more adverse events during the study.

There were no statistically significant differences in the overall incidence rates of postoperative cardiovascular complications between the high-dose aprotinin group $(61 \%)$, the low-dose aprotinin group (53\%), and the placebo group $(61 \%)$ when all reported events affecting the cardiovascular system were combined ( $p=0.565$ ).

There were no differences in the frequency of thrombotic events in patients treated with aprotinin or placebo, including thrombosis (high-dose aprotinin, 1\%; low-dose aprotinin, $0 \%$; placebo, $0 \%$ ) and deep vein thrombosis (high-dose aprotinin, 1\%; low-dose aprotinin, $0 \%$; placebo, $0 \%$ ). Myocardial infarctions were reported in four patients in the high-dose aprotinin group (6\%), one patient in the low-dose aprotinin group (1\%), and three patients in the placebo group $(4 \% ; p=0.54)$. Three patients were reported to have cerebrovascular accidents (one in each treatment group). One additional patient had a cerebral vascular accident (low-dose aprotinin) 31 days after discharge from the hospital.

Five patients died during the study (high-dose aprotinin, $n=3$; low-dose aprotinin, $n=2$ ). One patient in the low-dose aprotinin group died within 1 week of receiving the study medication. The 
Table V. Patients with renal dysfunction

\begin{tabular}{|c|c|c|c|c|c|}
\hline Treatment group & Status & Study ID & $\begin{array}{c}\text { Baseline creatinine } \\
\text { value }(\mathrm{mg} / \mathrm{dl})\end{array}$ & $\begin{array}{c}\text { Peak creatinine } \\
\text { value }(m g / d l)\end{array}$ & Days postop. \\
\hline \multirow[t]{8}{*}{ High-dose } & Diabetes & 1016 & 1.2 & 4.7 & 25 \\
\hline & Diabetes & 1021 & 1.3 & 4.1 & 7 \\
\hline & Diabetes & 5025 & 1.2 & 1.8 & 2 \\
\hline & No diabetes & 1040 & 0.9 & 2.5 & 16 \\
\hline & No diabetes & 3031 & 1.3 & 2.4 & 3 \\
\hline & No diabetes & 3038 & 1.6 & 2.3 & 2 \\
\hline & No diabetes & 3041 & 1.3 & 3.4 & 4 \\
\hline & No diabetes & 5040 & 0.9 & 4.3 & 7 \\
\hline \multirow[t]{5}{*}{ Low-dose } & No diabetes & 1002 & 1.8 & 3.7 & 7 \\
\hline & No diabetes & 1008 & 0.8 & 4.9 & 5 \\
\hline & No diabetes & 1035 & 1.3 & 3.2 & 36 \\
\hline & No diabetes & 2028 & 1.4 & 4.1 & 2 \\
\hline & No diabetes & 3028 & 1.4 & 8.1 & 5 \\
\hline
\end{tabular}

remainder of the patients died more than 1 month after receiving the study medication. One additional patient in the low-dose aprotinin group died during a hospital readmission 31 days after discharge.

Renal dysfunction. Renal dysfunction was significantly more prevalent in the high-dose $(11 \%)$ and low-dose $(7 \%)$ aprotinin groups than in the placebo group (0\%; $p=0.008)$. Table IV contains the breakdown of the reported renal treatment emergency events by study group.

The patients did not require dialysis, except for three (high-dose aprotinin, $n=1$; low-dose aprotinin, $n=2$ ) categorized as having renal dysfunction. Two (low-dose aprotinin) of the three patients who had renal failure necessitating dialysis died (sepsis and cardiogenic shock) without their renal dysfunction being resolved. In the third patient who required dialysis, the renal failure had resolved by the time of discharge.

To investigate whether certain baseline characteristics would correlate with the prevalence of renal dysfunction, we calculated by-treatment rates for the following factors: weight ( $\geq 85 \mathrm{~kg}$ vs $<85 \mathrm{~kg}$ ), sex, age $(\geq 60$ years vs $<60$ years), presence of diabetes mellitus, hypertension, congestive heart failure, aminoglycoside treatment, preoperative angiotensin-converting enzyme inhibitor, and transfusion of allogeneic blood ( $\geq 3$ units vs $<3$ units). Sixteen percent $(3 / 18)$ of the patients with diabetes mellitus had renal dysfunction in comparison with $5 \%(10 / 194)$ of those without diabetes (Table V). All three of the patients with diabetes in whom renal dysfunction developed were randomized to the highdose aprotinin group. However, interpretation of these data must take into account that the study comprised few patients with diabetes mellitus. Other factors associated with an increased incidence of renal events were age 60 years or older, a baseline diagnosis of congestive heart failure, transfusion of three or more units of allogeneic blood, aminoglycoside treatment, and preoperative treatment with angiotensin-converting enzyme inhibitors. No other trends in the incidence of renal dysfunction by baseline characteristics were observed.

Elevated baseline serum creatinine values (defined as a serum creatinine at any study site that exceeded the upper limit of the normal range at that site's laboratory) did not appear to predispose patients to renal dysfunction. In patients with an elevated baseline creatinine value, none of the patients treated with high-dose aprotinin, $17 \%$ of those treated with low-dose aprotinin, and none of those treated with placebo had postoperative renal dysfunction. The frequency of renal dysfunction in patients without an elevated baseline creatinine value was $12 \%$ in the high-dose aprotinin group, $6 \%$ in the low-dose aprotinin group, and $0 \%$ in the placebo group.

In view of the increased incidence of renal dysfunction in the high-dose aprotinin group, the incidence of a clinically significant increase in serum creatinine from the baseline preoperative sample to the postoperative samples was examined. Increases in creatinine concentration of $0.5 \mathrm{mg} / \mathrm{dl}$ or greater occurred in $30 \%(21 / 71)$ of the high-dose aprotinin group, $14 \%(10 / 70)$ of the low-dose aprotinin group, and $8 \%(6 / 71)$ of the placebo group $(p=0.003)$. This difference between the treatment groups did not persist when an increase in the serum creatinine of greater than $2.0 \mathrm{mg} / \mathrm{dl}$ was considered (Fig. 3).

Additional operations. Patients returned to the operating room for a variety of procedures including sternal débridement $(n=1)$, wound closure and intraaortic balloon pump removal $(n=1)$, bleeding 


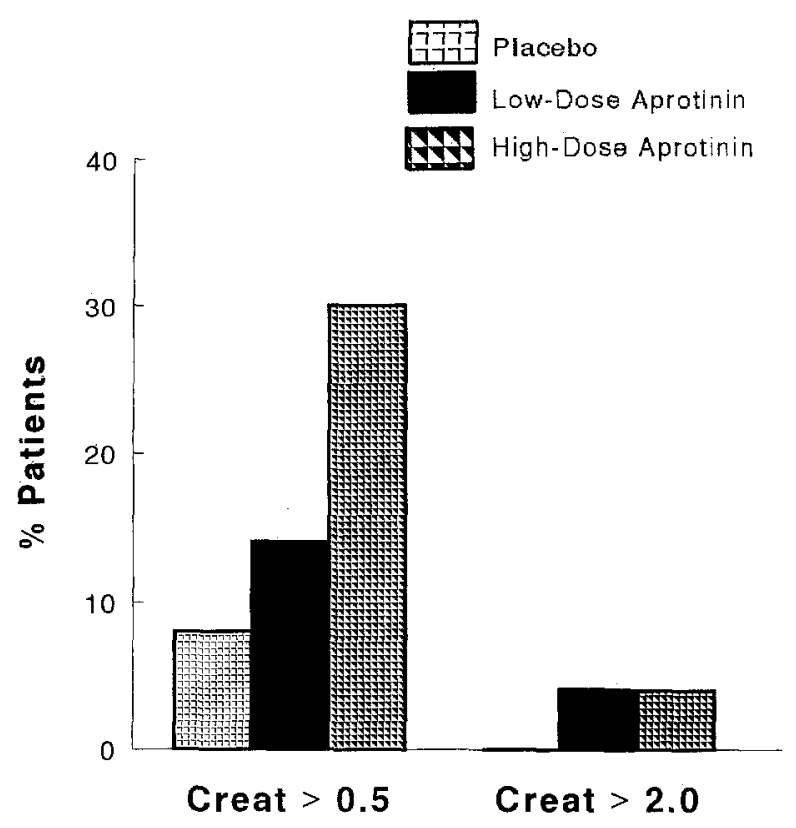

Fig. 3. Changes in serum creatinine concentration of $\geq 0.5 \mathrm{mg} / \mathrm{dl}$ occurred significantly more often in the aprotinin-treated group ( $p=0.003$ ). This difference did not persist for a change in creatinine of $>2 \mathrm{mg} / \mathrm{dl}$. Creat, Creatinine.

from a surgically localized anatomic site $(n=10)$, heart transplantation $(n=1)$, diffuse bleeding $(n=$ $1)$, perivalvular leak $(n=1)$, and insertion of a pacemaker $(n=2)$. Seven percent of the placebo group required an additional operation, as did 1\% of the high-dose aprotinin and low-dose aprotinin treatment groups ( $p=0.043$ for high- and low-dose aprotinin groups vs placebo).

\section{Discussion and conclusions}

According to previous reports, aprotinin reduces bleeding, decreases transfusion requirements, and is relatively safe when compared with other interventions used routinely in patients having cardiac operations. ${ }^{13}$ In the current study aprotinin was not effective in decreasing the transfusion requirements, probably because of the nature of the operations: primary sternotomy for valve operations, no leg incision, and short cardiopulmonary bypass and crossclamp times. Although there was no statistically significant difference in the transfusion rates for aprotinin-treated groups in comparison with the placebo group, the higher transfusion rate and smaller preoperative red cell volume seen in the high-dose aprotinin treatment group may have achieved statistical significance if the sample size had been larger. This would have supported the increased transfusion rate resulting from smaller preoperative red cell volumes.

The mechanism by which aprotinin reduces blood loss in patients undergoing cardiac operations appears to be through its ability to preserve normal coagulation. Aprotinin has been shown to inhibit contact activation, preserve the integrity of platelet receptors, ${ }^{15}$ inhibit fibrinolysis by binding reversibly to plasmin, ${ }^{6}$ inhibit various enzymes along the intrinsic clotting pathway, ${ }^{16}$ and block the inflammatory response by inhibiting the activation of prekallikrein. ${ }^{17}$ The use of a nonkallikrein-inhibiting aprotinin dose, the low-dose aprotinin regimen, in this study calls into question the latter mechanism of action, because a dose-dependent reduction in mediastinal drainage was seen.

A significant number of patients in the high-dose aprotinin group had a postoperative increase in serum creatinine of $\geq 0.5 \mathrm{mg} / \mathrm{dl}$. The selection of a $0.5 \mathrm{mg} / \mathrm{dl}$ change was based on the data previously reported in the literature. ${ }^{18}$ Despite the presence of statistically significant increases in the creatinine concentration, the development of postoperative renal dysfunction did not appear to have long-term clinical implications except in the three patients who required dialysis. Although the number of patients with diabetes participating in this study was small $(n=18)$, all of those who had renal failure were in the high-dose aprotinin group (3/8); renal failure did not develop in any of the patients with diabetes in the other two groups (low-dose aprotinin, 0/6; placebo, 0/4). This finding is consistent with the report by Lemmer and associates, ${ }^{19}$ in which patients with diabetes who were treated with aprotinin had increases in serum creatinine levels $(0.5 \mathrm{gm} / \mathrm{dl}$ or greater) more often than those randomized to the placebo group. Because the number of patients with diabetes in the present study was small, the finding requires further characterization with the establishment of prospective definitions of renal dysfunction. It is not an unexpected finding, however, considering the mechanism by which the kidney handles aprotinin. The proximal tubular epithelial cells take up small proteins like aprotinin and insulin to recycle the amino acids. With large protein loads, such as with a standard high-dose aprotinin treatment regimen, this system might be overwhelmed. Proximal tubular cells swell and mechanical effects on filtrate flow have been observed. As the cells process the protein over a 12 - to 24 -hour period, the mechanical effects reverse. ${ }^{19}$ 
Concern has been raised about the potential increased risk of thrombotic complications with aprotinin therapy in patients having cardiac operations. ${ }^{20}$ In this study, the rates of thrombotic complications did not differ among treatment groups.

The lack of efficacy may have been influenced by the lower red cell mass seen in the high-dose aprotinin group. Because there were no differences in the baseline hemoglobin values, the need for only one unit of allogeneic blood suggests that the transfusions in the high-dose aprotinin group were for inadequate red cell mass, not blood loss. The lack of correlation between mediastinal drainage and red cell transfusion in the high-dose aprotinin group also supports the conclusion that many transfusions in this group were the result of preexisting low red cell mass. In addition, the relative polycythemia seen in the high-dose group at discharge in comparison with the low-dose aprotinin and placebo groups suggests excessive transfusion. It is possible that anticipatory transfusion may be responsible for the higher transfusion rates seen in the high-dose aprotinin group which, when combined with the reduction in postoperative blood loss, translated into a significantly higher hematocrit value on discharge. This fact implies that, for whatever reason, the high-dose aprotinin group received excessive transfusions despite the fact that they bled less. This observation supports the need to include preoperative red cell mass in the randomization schemes in future studies of blood-sparing agents. It also suggests that using the number of patients receiving transfusions as a study end point is confounded by the problem of hemodilution, not just bleeding. Nonetheless, the data suggest that aprotinin should be used cautiously in patients with preoperative renal dysfunction or diabetes until further controlled studies can be conducted in these populations.

We recognize Dr. Alan Zaslavsky, Dr. Lawrence Schwartz, and Dr. Andrea Nadel for their help with the statistical analysis and the late Dr. Edwin Land and Mrs. Edwin Land for their kindness and moral support throughout the conduct of this study.

\section{REFERENCES}

1. Woodman RC, Harker LA. Bleeding complications associated with cardiopulmonary bypass. Blood 1990;76:168097.

2. Hardy JF, Perrault J, Tremblay N, Robitaille D, Blain R, Carrier $M$. The stratification of cardiac surgical procedures according to use of blood products: a retrospective analysis of 1480 cases. Can J Anaesth 1991;38:511-7.

3. Arom KV, Emery RW. Decreased postoperative drainage with addition of epsilon-aminocaproic acid before cardiopulmonary bypass. Ann Thorac Surg 1994;57:1108-112.

4. Murase M, Usui A, Tomita Y, Maeda M, Koyama T, Abe T. Nafamostat mesilate reduces blood loss during open heart surgery. Circulation 1993;88(5 Pt 2):II432-6.

5. Karski JM, Teasdale SJ, Norman PH, Carroll JA, Weisel RD, Glynn MF. Prevention of postbypass bleeding with tranexamic acid and epsilon-aminocaproic acid. J Cardiothorac Vasc Anesth 1993;7:410-5.

6. Fritz H, Wunderer G. Biochemistry and applications of aprotinin, the kallikrein inhibitor of bovine organs. Arzneimittelforsch 1983;33:479-94.

7. Royston D, Bidstrup BP, Taylor KM, Sapsford RN. Effect of aprotinin on need for blood transfusion after repeat openheart surgery. Lancet 1987;2:1289-91.

8. Baden H, Jordal K, Lund F, Zachariae F. Prophylactic and curative action of Trasylol in pancreatitis: a double-blind trial. Scand J Gastroenterol 1968;4:291-5.

9. Glenn TM, Herlich BL, Lefer AM. Protective action of a protease inhibitor in haemorrhagic shock. Arch Int Pharmacodyn Ther 1973;203:292-304.

10. McMichan JC, Rosengarten DS, Philipp E. Prophylaxis of post-traumatic pulmonary insufficiency by protease inhibitor therapy with aprotinin: a clinical study. Circ Shock 1982;9: 107-16.

11. Schneider B, Schnells G, Trentz O, Tscherne H. Field study on the therapeutic value of Trasylol in traumatic shock. Rev Int Serv Sante Armees Terr Mes Air 1979;52:251-6.

12. Kirkin JK, Westaby S, Blackstone EH, Kirklin JW, Chenoweth DE, Pacifico AD. Complement and the damaging effects of cardiopulmonary bypass. J Thorac Cardiovasc Surg $1983 ; 86: 845-57$.

13. Royston D. High-dose aprotinin therapy: a review of the first five years' experience. J Cardiothorac Vasc Anesth 1992;6: $76-100$.

14. Medtronic Hemotec Inc. Hepcon HMS Operators Manual. 1989.

15. Lavee J, Savion N, Smolinsky A, Goor DA, Mohr R. Platelet protection by aprotinin in cardiopulmonary bypass: electron microscopic study. Ann Thorac Surg 1992;53:477-81.

16. Dubber AHC, McNicol GP, Uttley D, Douglas AS. In vitro and in vivo studies with Trasylol, an anticoagulant and fibrinolytic inhibitor. Br J Haematol 1968;14:31-49.

17. Hoffmann $H$, Siebeck M, Thetter O, Jochum M, Fritz $H$. Aprotinin concentrations effective for the inhibition of tissue kallikrein and plasma kallikrein in vitro and in vivo. Adv Exp Med Biol 1989;247B:35-42.

18. Salzman EW, Weinstein MJ, Weintraub RM, et al. Treatment with desmopressin acetate to reduce blood loss after cardiac surgery: a double-blind randomized trial. $N$ Engl J Med 1988;314:1402-6.

19. Lemmer JH, Stanford W, Bonney SL, Chomka EV, Karp RB, Laub GW, et al. Aprotinin for coronary artery bypass grafting: effect on postoperative renal function. Ann Thorac Surg 1995;59:132-6.

20. Cosgrove DM, Heric B, Lytle BW, Taylor PC, Novoa R, Golding LAR, et al. Aprotinin therapy for reoperative myocardial revascularization: a placebo-controlled study. Ann Thorac Surg 1992;54:1031-8. 